\title{
X-linked inhibitor of apoptosis (XIAP) and XIAP-associated factor-1 expressions and their relationship to apoptosis in human hepatocellular carcinoma and non-cancerous liver tissues
}

\author{
RYOSUKE SAKEMI ${ }^{1-3}$, HIROHISA YANO ${ }^{1,3}$, SACHIKO OGASAWARA ${ }^{1,3}$, JUN AKIBA ${ }^{1,3}$, \\ OSAMU NAKASHIMA ${ }^{1,3}$, SUGURU FUKAHORI ${ }^{1,3}$, MICHIO SATA ${ }^{2,3}$ and MASAMICHI KOJIRO ${ }^{1,3}$ \\ ${ }^{1}$ Department of Pathology and ${ }^{2}$ Division of Gastroenterology, Department of Medicine, Kurume University School \\ of Medicine; ${ }^{3}$ Research Center of Innovative Cancer Therapy of the 21st Century COE Program \\ for Medical Science, Kurume University, Kurume, Fukuoka 830-0011, Japan
}

Received March 1, 2007; Accepted April 2, 2007

\begin{abstract}
The X-linked inhibitor of apoptosis (XIAP) belongs to the inhibitor of apoptosis (IAP) family, and the action of XIAP is inhibited by XIAP-associated factor-1 (XAF1). In the present study, XIAP and XAF1 protein expressions and their relationship to apoptosis were investigated in hepatocellular carcinoma (HCC). We examined immunohistochemical expressions of XIAP and XAF1, and the number of apoptotic HCC cells in surgically resected tissues of $24 \mathrm{HCCs}$, consisting of 7 well-, 10 moderately and 7 poorly differentiated HCCs. As a result, XIAP and XAF1 expressions were identified in the cytoplasm of non-neoplastic and neoplastic hepatocytes. In the 24 HCCs, XIAP expression was not different according to the histological grade of HCC. In contrast, XAF1 expression was significantly lower in poorly differentiated than that in well- or moderately differentiated HCCs $(\mathrm{P}=0.001)$, or XIAP expression in poorly differentiated $\mathrm{HCC}(\mathrm{P}<0.001)$. Apoptotic HCC cell number was significantly lower in poorly differentiated than that in well- or moderately differentiated HCCs $(\mathrm{P}<0.01)$. A significant relationship was observed between XAF1 expression and apoptotic cell number in HCC tissues. In conclusion, the present findings suggest that significantly low XAF1 expression, but not XIAP expression, in poorly differentiated HCC may relate to resistance to apoptosis.
\end{abstract}

\section{Introduction}

Apoptosis is an active process of gene-directed cellular selfdestruction and is observed in the sculpting of organs and tissues during embryonic development and the removal of old

Correspondence to: Dr Hirohisa Yano, Department of Pathology, Kurume University School of Medicine, 67 Asahi-machi, Kurume, Fukuoka 830-0011, Japan

E-mail: hiroyano@med.kurume-u.ac.jp

Key words: apoptosis, hepatocellular carcinoma, XIAP-associated factor-1, X-linked inhibitor of apoptosis unnecessary cells for the maintenance of tissue homeostasis. Apoptosis is also observed in some pathological processes, such as in the elimination of tumor cells and virus-infected cells, and thereby contributes to the self-defense mechanisms. In the process of apoptosis, activation of the caspase family, especially caspase-3, plays an important role (1-3).

The inhibitor of apoptosis (IAP) family contains intrinsic cellular regulators of apoptosis and includes X-linked IAP (XIAP), c-IAP1, c-IAP2, NAIP, ML-IAP, ILP-2, survivin and Apollon (4). These proteins are characterized by the presence of one to 3 copies of a $\sim 70$-amino acid domain termed the baculoviral inhibitory repeat (BIR) at the amino terminus of the protein. The BIR domains have been shown to bind and inhibit caspase-3, -7, and -9 (4-6). Among the IAP family, XIAP protein contains 3 copies of the BIR domain and one RING domain at the extreme carboxyl terminus of the protein. In vitro kinetic studies have shown that XIAP is the most potent caspase inhibitor and suppressor of apoptosis in the IAP family $(4,6)$. Various levels of XIAP mRNA and protein were expressed in human cancer cell lines $(7,8)$, suggesting the involvement of XIAP in the apoptosis resistance mechanism of cancer cells.

The caspase-inhibiting activity of XIAP is negatively regulated by at least two XIAP-interacting proteins, XIAPassociated factor-1 (XAF1) $(8,9)$ and Smac/DIABLO $(10,11)$. $\mathrm{XAF} 1$, a 34-kDa zinc finger protein, was identified in a yeast two-hybrid screen based on its ability to bind XIAP and was found to antagonize the ability of XIAP to suppress caspase activity and cell death in vitro (6). XAF1, which resides in the nucleus, can effect a relocalization of XIAP from the cytoplasm to nucleus and neutralize XIAP's ability to inhibit apoptosis (9). Smac/DIABLO, which is localized in mitochondria, is released into cytoplasm and processed into an active form during mitochondria-induced apoptosis $(10,11)$. The binding of active Smac/DIABLO to XIAP is proposed to destabilize the XIAP-caspase interaction by steric hindrance, resulting in disruption of the XIAP-caspase complex $(12,13)$. $\mathrm{XAF} 1$ is ubiquitously expressed in normal tissues, but is present at very low or undetectable levels in many cancer cell lines $(8,9)$. Byun et al (14) found that a substantial fraction of gastric cancer cell lines and tissues express no or extremely low levels 
of the XAF1 transcript, whereas Smac/DIABLO was normally expressed in all cancer specimens.

Hepatocellular carcinoma (HCC) often develops in patients with hepatitis $\mathrm{B}$ or $\mathrm{C}$ virus-related chronic hepatitis or liver cirrhosis. In non-HCC tissues, apoptosis of hepatocytes, showing chromatin condensation of the nuclei and eosinophilic change of the cytoplasm, is often observed, and the number of such apoptotic cells is in proportion to inflammatory activity. On the other hand, abnormalities in the expression of apoptosisrelated molecules, and the resistance to various apoptosisinducing stimuli have been reported in $\operatorname{HCC}(15,16)$. Except for the study of Shiraki et al (17) who examined XIAP expression in HCC tissues, there have been no comparative studies on XAF1 and XIAP expressions according to the histological grade, and no study to explore the relationship between the frequency of apoptosis and XAF1 or XIAP expression in human HCC tissues. These are addressed in the present study.

\section{Materials and methods}

Tissue samples. Immunohistochemical examination was performed on formalin-fixed, paraffin-embedded sections of cancerous and non-cancerous tissues obtained from 24 surgically resected HCCs at the Kurume University Hospital between 1989 and 2003. The 24 patients ranged from 50 to 84 years of age, and consisted of 18 males and 6 females. Two were serum hepatitis B surface antigen-positive, 19 were positive to the hepatitis $\mathrm{C}$ virus antibody, and the remaining 3 patients were not positive to either. The 24 cases did not receive preoperative anticancer therapies such as transcatheter arterial embolization (TAE) and percutaneous ethanol injection therapy. Among the 24 cases, seven had well-differentiated HCC, 10 had moderately differentiated HCC, and 7 had poorly differentiated HCC. The non-HCC tissues showed liver cirrhosis in ten cases and chronic hepatitis in 14. Informed consent was obtained from all patients included in the study.

Immunohistochemistry. Formalin-fixed, paraffin-embedded serial sections $(4 \mu \mathrm{m})$ were mounted on 3-aminopropyltriethoxysilane-coated slides (Matsunami Glass Ind., Ltd., Osaka, Japan), and deparaffinized in xylene alcohol and graded alcohol. For XIAP immunostaining, the sections were soaked in $10 \mathrm{mmol} / \mathrm{l}$ of sodium citrate buffer $(\mathrm{pH}$ 6.9) and treated in a microwave for $30 \mathrm{~min}$ for antigen retrieval. XIAP and XAF1 expressions were immunohistochemically examined with mouse monoclonal anti-XIAP antibody $(3.0 \mu \mathrm{g} / \mathrm{ml}, \mathrm{BD}$ Biosciences, San Jose, CA) and rabbit polyclonal anti-XAF1 antibody ( $4 \mu \mathrm{g} / \mathrm{ml}$, IMGENEX, San Diego, CA) as the primary antibodies, and using catalyzed signal-amplification system II (Code K1497, Dako, Ely, UK) according to the manufacturer's protocol. The sections for XIAP immunostaining were incubated with primary antibody for $60 \mathrm{~min}$ at room temperature after blocking endogenous biotin and peroxidase activities, and the sections for XAF1 were incubated overnight with primary antibodies at $4^{\circ} \mathrm{C}$. Negative controls were prepared by replacing the primary antibody with normal mouse IgG or normal rabbit IgG. The peroxidase reaction was developed with the addition of 3,3-diaminobenzidine and $\mathrm{H}_{2} \mathrm{O}_{2}$ substrate solution. After counterstaining with hematoxylin, the slides were dehydrated, coverslipped, and observed under a microscope (Olympus BH-2, Olympus Optical, Tokyo, Japan).

Evaluation of immunohistochemical findings. The results of immunohistochemistry were evaluated according to the rate of staining and grading of expression by two pathologists (Y.S. and H.Y.). XIAP and XAF1 expressions in non-HCC tissues are relatively homogeneous and were used as an internal positive control. Regarding XIAP and XAF1, an expression score system was assigned on the basis of multiplying the rate of cells staining positive by the intensity of staining. The staining intensity was scored on a scale from 0 to $2(0, \mathrm{HCC}$ cells with no positive reactions; 0.5 , HCC cells stained less intensely than hepatocytes; 1.0, HCC cells stained as intensely as hepatocytes; 2.0, HCC cells more intensely stained than hepatocytes). The final score was calculated as the sum of each staining intensity multiplied by the rate of the corresponding area. For example: if a HCC nodule shows $30 \%$ HCC cells stained less intensely than hepatocytes, 50\% HCC cells stained as intensely as hepatocytes, and 20\% HCC cells more intensely stained than hepatocytes, the score would be $(0.3 \times 0.5)+(0.5 \times 1.0)+(0.2 \times 2.0)=1.05$.

Assessment of number of apoptosic cells in HCC tissues. The number of cells showing the characteristics of apoptosis (e.g., cytoplasmic shrinkage, chromatin condensation and nuclear fragmentation) was counted in 14-25 $0.25 \mathrm{~mm}^{2}$-areas within HCC nodules stained with hematoxylin-eosin (HE).

Statistics. Group differences were obtained for the expression score of XIAP and XAF1, and apoptosis number with the Mann-Whitney test. The correlation between the number of apoptosic cells and the expression of XIAP or XAF1 was examined by Pearson's correlation coefficient. All statistical analyses were performed with StatMate III (ATMS Co., Ltd., Tokyo, Japan). P-values $<0.05$ were considered significant.

\section{Results}

XIAP and XAF1 expressions in HCC and non-HCC tissues. In non-HCC tissue, XIAP was expressed in the cytoplasm of hepatocytes, and the XIAP-expressing cells were relatively homogeneously distributed in the liver lobule (Fig. 1A and B). XAF1 was also expressed in the cytoplasm of hepatocytes. The XAF1-expressing cells were almost homogeneously distributed, but the more strongly expressing cells were scattered in the areas around portal tracts with marked cellular infiltration (Fig. 1C). Cirrhosis and chronic hepatis did not differ in the intensity or distribution of XAF1 expression. Negative controls showed no staining for XIAP or XAF1 (data not shown).

In HCC tissue, XIAP was expressed in the cytoplasm of HCC cells, and the XIAP-expressing cells in cancer nodules were more homogeneously distributed than the XAF1expressing cells (Fig. 2A and B). XAF1 was also expressed in the cytoplasm of HCC cells. However, the XAF1 expression levels in well- and moderately differentiated HCC nodules varied with individual cancer cells, showing a heterogeneous distribution. In particular, XAF1 expression was conspicuous in $\mathrm{HCC}$ cells with fatty change and immediately subcapsular HCC cells in the periphery of cancer nodules (Fig. 2A and B; Fig. 3). 


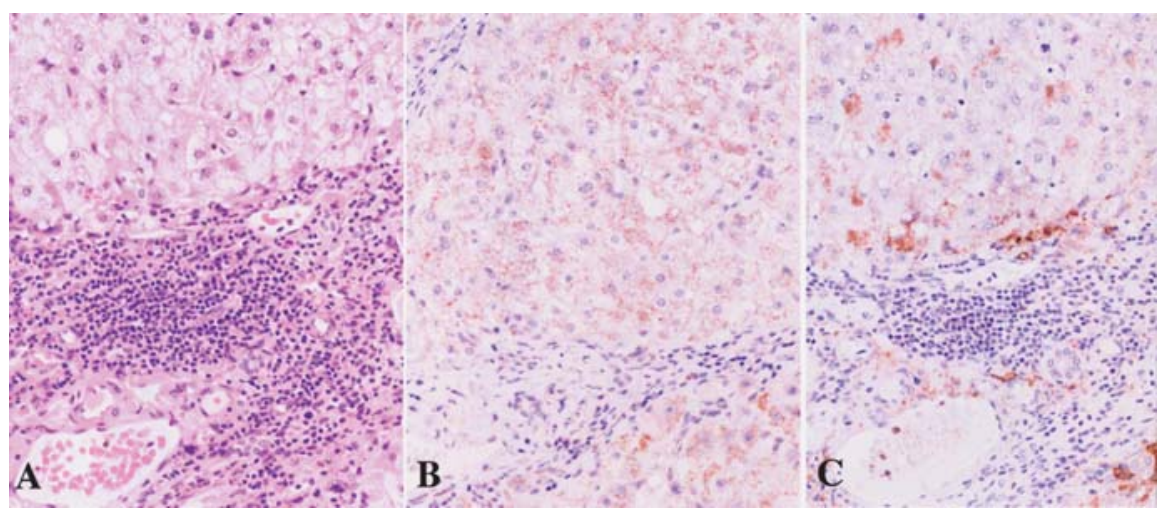

Figure 1. (A) Photomicrograph showing a portal area with active infiltration of lymphocytes, and periportal non-neoplastic hepatocytes (hematoxylin-eosin stain, x100). (B) Immunohistochemical staining for XIAP showing homogeneous expression in hepatocytes (counterstained with Mayer's hematoxylin, x100). (C) Immunohistochemical staining for XAF1 showing homogeneous expression in hepatocytes, except in those around the portal areas with active infiltration of inflammatory cells, which showed strong expression (counterstained with Mayer's hematoxylin, x100).

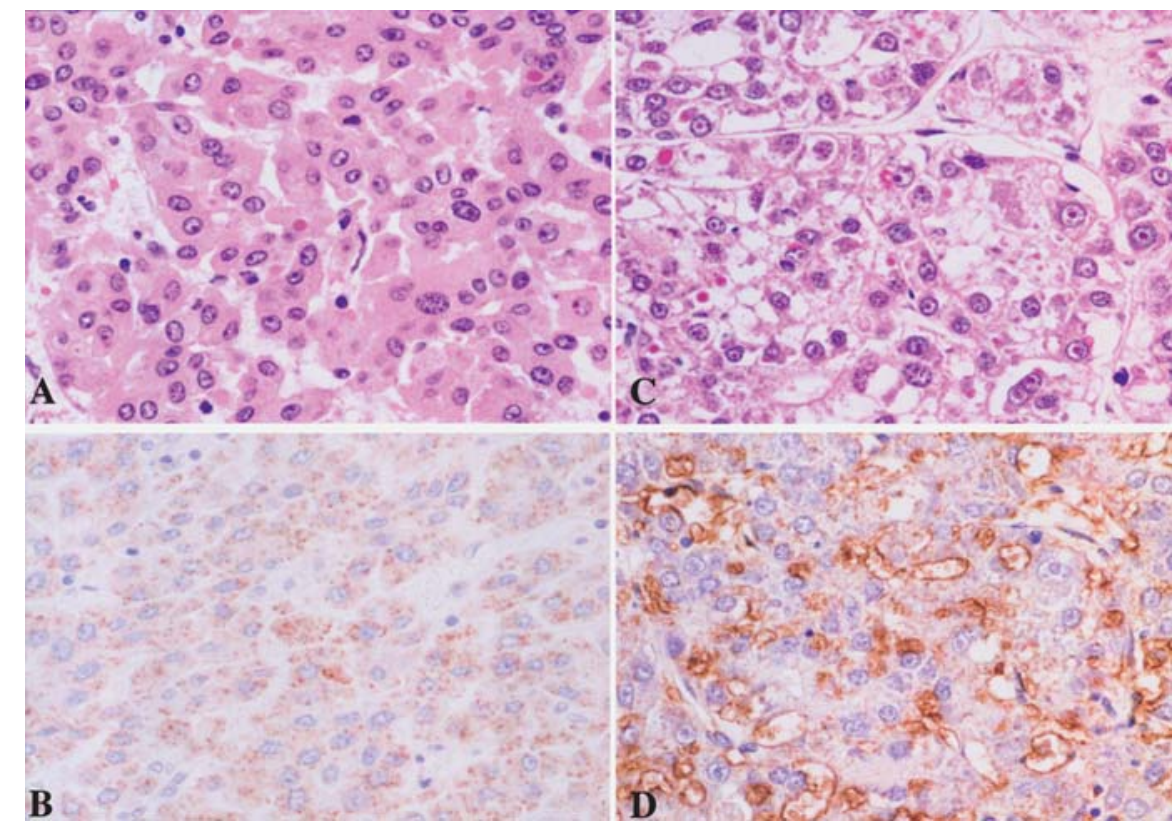

Figure 2. (A) Photomicrograph showing moderately differentiated hepatocellular carcinoma (HCC) with a thick trabecular arrangement (hematoxylin-eosin stain, x200). (B) Immunohistochemical staining for XIAP showing homogeneous expression in HCC cells (counterstained with Mayer's hematoxylin, x200). (C) Photomicrograph showing moderately differentiated HCC with fatty change and a relatively compact arrangement (hematoxylin-eosin stain, $\mathrm{x} 200$ ). (D) Immunohistochemical staining for XAF1 showing strong expression in HCC cells with fatty change (counterstained with Mayer's hematoxylin, x200).

The numbers of well-, moderately and poorly differentiated HCCs with an XIAP expression score of 1 or higher were 5 $(71 \%), 5(50 \%)$ and $6(86 \%)$, respectively. The XIAP expression scores in the well-, moderately and poorly differentiated HCCs were $1.07 \pm 0.44$ (mean \pm SD), $1.10 \pm 0.69$ and $1.17 \pm 0.38$, respectively, showing no significant differentiation-dependent differences [Fig. 4 (left panel)]. The numbers of well-, moderately and poorly differentiated HCCs with an XAF1 expression score of 1 or higher were $6(86 \%), 6(60 \%)$ and $0(0 \%)$, respectively. The XAF1 expression scores in the well-, moderately and poorly differentiated HCCs were $1.14 \pm 0.54$ (mean \pm SD), $1.14 \pm 0.47$ and $0.19 \pm 0.16$, respectively, indicating that the expression was significantly lower in the poorly differentiated HCCs than in the well- and moderately differentiated HCCs $[\mathrm{P}<0.001$, Fig. 4 (left panel)]. In the poorly differentiated HCCs, the expression score of XAF1 was significantly lower than that of XIAP $[\mathrm{P}<0.001$, Fig. 4 (right panel)]. No other differentiation-dependent differences were noted between the expression scores of XIAP and XAF1.

Presence of apoptosis and its relationship to XIAP and XAF1 expression in HCC. Fig. 5A shows typical apoptotic tumor cells (arrows) in moderately differentiated HCC tissue. The numbers of apoptotic cells per area in well-, moderately and poorly differentiated HCC nodules were $3.55 \pm 1.86$ (mean \pm SD), $3.62 \pm 1.46$ and $1.76 \pm 0.46$, respectively, indicating that the number of apoptotic cells was significantly smaller in the poorly differentiated than in the well- and moderately differentiated HCCs $(\mathrm{P}<0.01$, Fig. 5B). In the $24 \mathrm{HCCs}$, the number of apoptotic cells per area was significantly correlated with XAF1 expression, but not with XIAP expression (Fig. 6). 

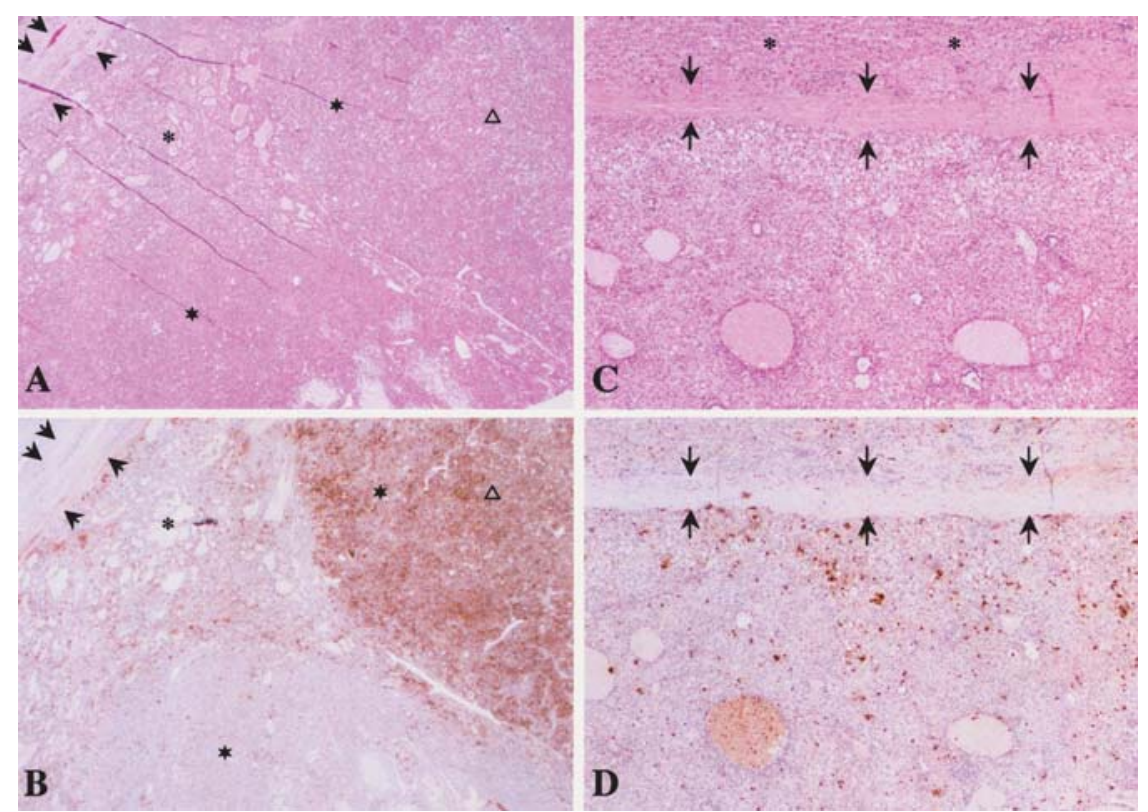

Figure 3. (A) Photomicrograph showing a hepatocellular carcinoma (HCC) nodule with a fibrous capsule (arrows). The nodule contains areas of moderately differentiated HCC with a trabecular arrangement $(*)$, that with a pseudoglandular arrangement $(*)$, and that with fatty change $(\Delta)$ (hematoxylin-eosin stain, $\mathrm{x} 5)$. (B) Immunohistochemical staining for XAF1 showing heterogeneous expression. Upper right areas of moderately differentiated HCC with fatty change $(\Delta)$ and surrounding moderately differentiated HCC show strong expression, whereas the other areas show low levels of expression (counterstained with Mayer's hematoxylin, x5). (C) Photomicrograph showing a HCC nodule with a fibrous capsule (arrows) surrounded by non-HCC tissues (*) (hematoxylineosin stain, x20). (D) Immunohistochemical staining for XAF1 showing that HCC cells near the capsule (arrows) tend to show stronger XAF1 expression (counterstained with Mayer's hematoxylin, x20).

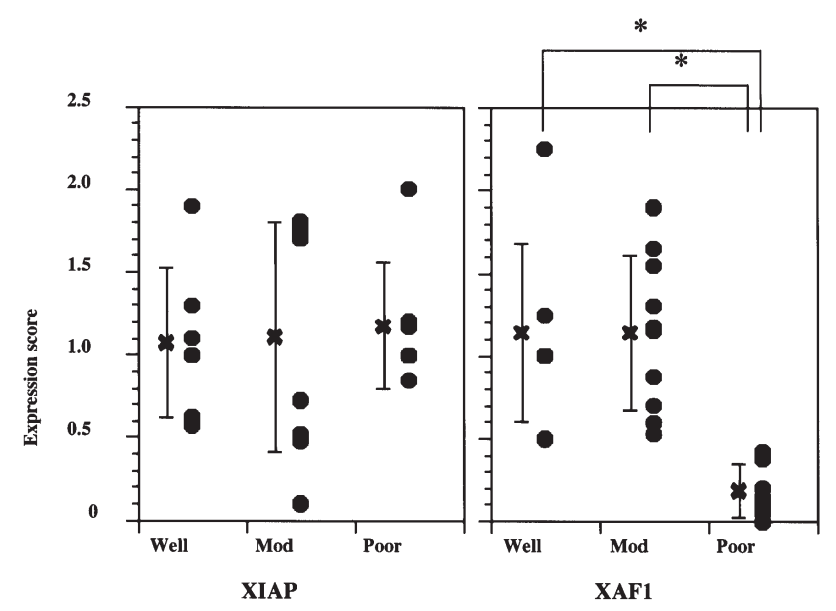

Figure 4. (Left panel) Expression scores of XIAP and XAF1 according to the histological grade of hepatocellular carcinoma (HCC). (Right panel) Comparison of expression scores between XIAP and XAF1 in poorly differentiated HCC. Data represent the mean \pm SD $(n=7-10)$. Well, welldifferentiated HCC; mod, moderately differentiated HCC; poor, poorly differentiated HCC. ${ }^{*} \mathrm{P}<0.001$ by the Mann-Whitney test.

\section{Discussion}

A recent immunohistochemical study revealed that the expression of XIAP protein in normal human tissues was heterogeneous and showed a higher selectivity to particular cell types (18). The expression of XIAP has also been confirmed in various tumor cell lines by Western blot analysis (7) and in various malignant neoplastic tissues, including non-small cell lung cancer, cervical carcinoma, prostate carcinoma and esophageal squamous cell carcinoma by immunohistochemistry,
A
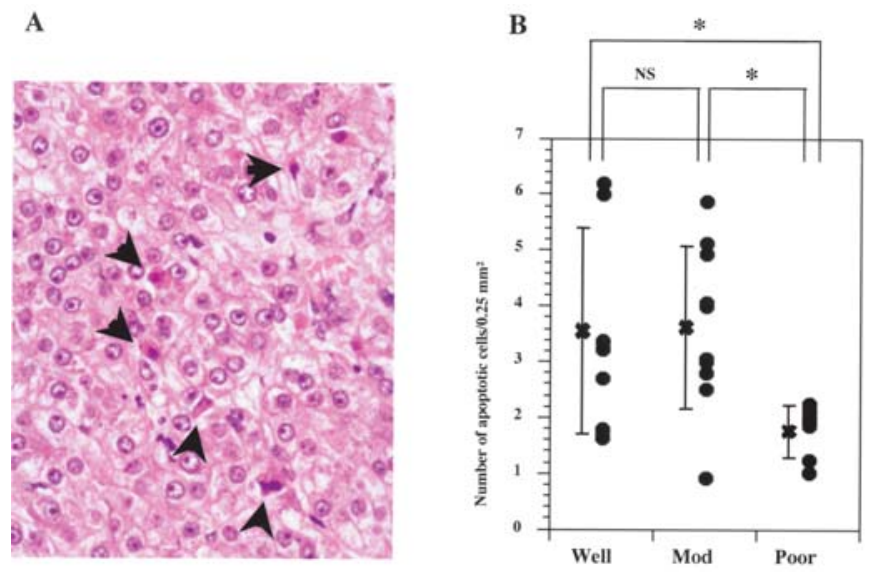

Figure 5. (A) Photomicrograph showing typical apoptotic tumor cells (arrows) with eosinophilic shrunken cytoplasm and pyknotic nuclei in moderately differentiated hepatocellular carcinoma (HCC) tissue (hematoxylineosin stain, x400). (B) Number of apoptotic HCC cells according to the histological grade. Data represent the mean \pm SD $(n=7-10)$. Well, welldifferentiated HCC; mod, moderately differentiated HCC; poor, poorly differentiated HCC; NS, not significant. ${ }^{*} \mathrm{P}<0.01$ by the Mann-Whitney $\mathrm{U}$ test.

in which a higher expression was observed compared with normal counterparts (reviewed in ref. 18). These results suggest that overexpression of XIAP may contribute to resistance to apoptosis in various types of cancer cells. Studies using immunostaining have reported that XIAP protein is absent or weakly expressed in normal liver and the non-cancerous tissue of HCC $(17,18)$. In contrast, Shiraki et al (17) reported that 14 of $20(70 \%)$ HCC tissue samples demonstrated moderate or strong cytoplasmic staining for XIAP, and that XIAP expression was inversely correlated with apoptosis in 


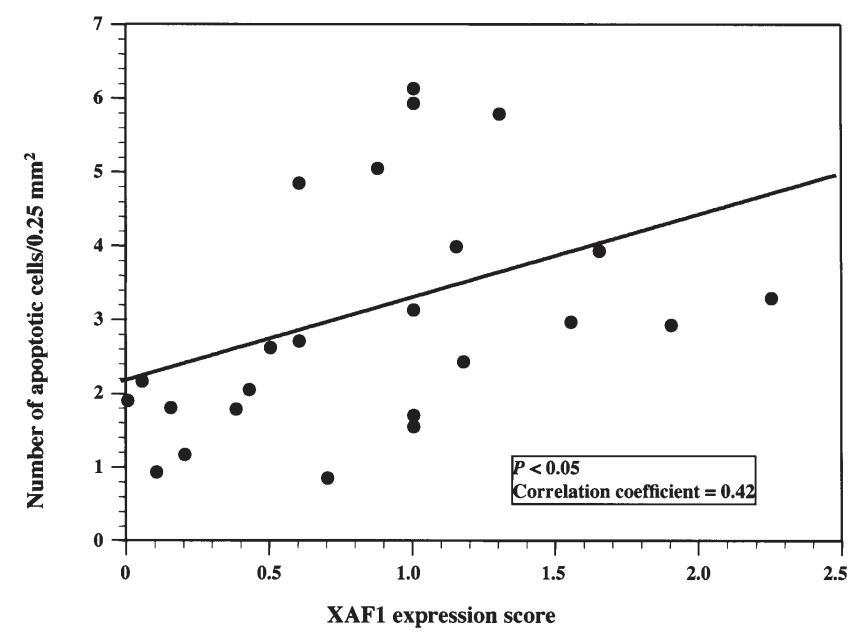

Figure 6. Relationship between the XAF1 expression score and the number of apoptotic tumor cells in hepatocellular carcinoma. Correlation of the coefficient rate is $0.42(\mathrm{P}<0.05)$.

HCC (17). In this study, HCC specimens with an XIAP expression score of 1 (indicating the same staining intensity in cancerous and non-cancerous tissues) or higher accounted for $16 / 24(67 \%)$, about half of which showed moderate to marked expression, with no significant differentiation-dependent differences [Fig. 4 (left panel)] or relationship between XIAP expression and cancer cell apoptosis.

We examined immunohistochemically the XAF1 protein expression in human HCC tissues and non-HCC tissues. Previous studies revealed that XAF1 mRNA is expressed ubiquitously in all normal adult and fetal tissues including the liver $(8,9)$. In non-HCC tissues, XAF1 was almost homogeneously expressed in non-neoplastic hepatocytes, except in those around the portal areas with active infiltration of inflammatory cells, which showed strong expression. Possible causes of this are: 1) The strong XAF1 expression could be mediated by inflammatory cytokines released from inflammatory cells, Leaman et al (19) found that XAF1 mRNA expression is upregulated by inflammatory cytokines, such as interferon (IFN)- $\gamma$ and tumor necrosis factor- $\alpha .2$ ) Strong XAF1 expression in periportal hepatocytes may be related with the progression of cytotoxic $\mathrm{T}$ lymphocyteinduced apoptosis, probably via the Fas/Fas ligand system (16).

In contrast to the normal tissues, XAF1 is present at very low or undetectable levels in a variety of cancer cell lines $(8,9)$, including melanoma (20), colorectal cancer (21), urinary bladder cancer, renal cancer and prostate cancer cell lines (22). In addition, XAF1 mRNA expression in melanoma tissues was significantly reduced compared with benign melanocytic nevi (20), and XAF1 mRNA in primary gastric tumors (14), and bladder transitional cell carcinoma and renal cell carcinoma tissues (22) were substantially lower compared with the non-cancerous tissue. Lee et al (22) found that hypermethylation at $14 \mathrm{CpG}$ sites in the 5' proximal region of the XAF1 promoter was highly prevalent in cancers versus adjacent normal or benign tissue and tightly associated with reduced gene expression. Nodules of HCC, particularly well- and moderately differentiated HCCs, were characterized by the heterogeneous (areas of high and low) expression of XAF1, with a tendency toward high expression in HCC cells with fatty change and in the periphery of cancer nodules. On the other hand, XAF1 expression was lower in the poorly differentiated than in the well- and moderately differentiated HCCs. Abnormal reduction of XAF1 mRNA which showed a good correlation with tumor grade, was also reported in gastric and bladder carcinomas $(14,22)$. We found a significant correlation between XAF1 expression and apoptosis; a significant reduction in apoptosis was observed in the poorly differentiated HCCs with a significantly lower expression of XAF1. It has been reported that the relative increase of XIAP to XAF1 expression may provide a survival advantage for tumor cells through the relative increase of XIAP antiapoptotic function (9). XAF1 inactivation in poorly differentiated HCC might contribute to the resistance to apoptosis and malignant progression of HCC. The causes of abnormal expression of XAF1 in HCC require further investigation.

Although this study immunohistochemically demonstrated the expression of XAF1 in the cytoplasm of neoplastic and nonneoplastic hepatocytes, endogenous XAF1 has been reported to be localized in the nucleus (8). However, XAF1 expression has been immunohistochemically detected in both the nucleus and cytoplasm of melanoma and benign nevus cells (20). In addition, its expression has also been noted in the cytoplasm and nucleus of XAF1-transfected 253J cells (22) and IFN- 3 stimulated A375 melanoma cells. Although it is not clear why XAF1 expression was demonstrable only in the cytoplasm in this study, we speculate that the causes of this are related to features specific to hepatocytes and the epitope accessibility of the antibody used. Therefore, further studies need to be performed using cell lines and different antibodies.

Type I IFN, including IFN- $\alpha$ and IFN- $\beta$, has various biologic functions, such as an antiproliferative action $(23,24)$. Recently, XAF1 was identified as an IFN-stimulated gene that contributes to IFN-dependent sensitization of cells to tumor necrosis factor-related apoptosis-inducing ligand-induced apoptosis (19). IFNs induced high levels of XAF1 protein predominantly in cell lines sensitive to the proapoptotic effects of IFN- $\beta$ (19). The direct antiproliferative effect of various type I IFN preparations and IFN- $\alpha$ subtypes on HCC cell lines has been reported (25-27) and upregulation of XAF1 mRNA following PEG-IFN- $\alpha 2 b$ treatment was also observed in the human HCC cell line HAK-1B (unpublished data). In clinical practice, IFN- $\alpha$ in combination with 5-fluorouracil (FU) has been used for the treatment of advanced HCCs, and the recent objective response rate of combination chemotherapy with IFN- $\alpha$ and 5-FU was 52\% among HCC patients with portal venous invasion (28). We speculate that XAF1 could be related with the susceptibility to the therapy, and HCCs with the loss or low levels of XAF1 could be more resistant to the combination therapy than HCCs with normal XAF1 expression. Further study is required to examine whether or not XAF1 expression could be a clinically useful marker for the prediction of the outcome of combination chemotherapy with IFN- $\alpha$ and 5-FU.

\section{Acknowledgements}

We thank Ms. Sachiyo Maeda and Misato Shiraishi for their assistance in our experiments. This study was supported in 
part by the Sarah Cousins Memorial Fund, Boston, MA, and by a Grant-in-Aid from the Ministry of Health, Labor and Welfare of Japan (No. 17200501).

\section{References}

1. Nagata S: Apoptosis by death factor. Cell 88: 355-365, 1997.

2. Vaux DL, Haecker G and Strasser A: An evolutionary perspective on apoptosis. Cell 76: 777-779, 1994.

3. Thompson CB: Apoptosis in the pathogenesis and treatment of disease. Science 267: 1456-1462, 1995.

4. Salvesen GS and Duckett CS: IAP proteins: blocking the road to death's door. Nat Rev Mol Cell Biol 3: 401-410, 2002

5. Deveraux QL, Takahashi R, Salvesen GS and Reed JC: X-linked IAP is a direct inhibitor of cell-death proteases. Nature 388: 300-304, 1997.

6. Holcik M, Gibson H and Korneluk RG: XIAP: apoptotic brake and promising therapeutic target. Apoptosis 6: 253-261, 2001.

7. Tamm I, Kornblau SM, Segall H, et al: Expression and prognostic significance of IAP-family genes in human cancers and myeloid leukemias. Clin Cancer Res 6: 1796-1803, 2000.

8. Fong WG, Liston P, Rajcan-Separovic E, St Jean M, Craig C and Korneluk RG: Expression and genetic analysis of XIAPassociated factor 1 (XAF1) in cancer cell lines. Genomics 70: 113-122, 2000.

9. Liston P, Fong WG, Kelly NL, et al: Identification of XAF1 as an antagonist of XIAP anti-Caspase activity. Nat Cell Biol 3: $128-133,2001$

10. Verhangen AM, Ekert PG, Pakusch M, et al: Identification of DIABLO, a mammalian protein that promotes apoptosis by binding to and antagonizing IAP proteins. Cell 102: 43-53, 2000.

11. Du C, Fang M, Li Y, Li L and Wang X: Smac, a mitochondrial protein that promotes cytochrome c-dependent caspase activation by eliminating IAP inhibition. Cell 102: 33-42, 2000.

12. Liu Z, Sun C, Olejniczak ET, et al: Structural basis for binding of Smac/DIABLO to the XIAP BIR3 domain. Nature 408: 1004-1008, 2000.

13. Wu G, Chai J, Suber TL, Wu JW, Du C, Wang X and Shi Y: Structural basis of IAP recognition by Smac/DIABLO. Nature 408: 1008-1012, 2000.

14. Byun DS, Cho K, Ryu BK, Lee MG, Kang MJ, Kim HR and Chi SG: Hypermethylation of XIAP-associated factor 1, a putative tumor suppressor gene from the 17p13.2 locus, in human gastric adenocarcinomas. Cancer Res 63: 7068-7075, 2003.
15. Yano H, Fukuda K, Haramaki M, Momosaki S, Ogasawara S, Higaki K and Kojiro M: Expression of Fas and anti-Fas-mediated apoptosis in human hepatocellular carcinoma cell lines. J Hepatol 25: 454-464, 1996.

16. Higaki K, Yano H and Kojiro M: Fas antigen expression and its relationship with apoptosis in human hepatocellular carcinoma and non-cancerous tissues. Am J Pathol 149: 429-437, 1996.

17. Shiraki K, Sugimoto K, Yamanaka Y, et al: Overexpression of $\mathrm{X}$-linked inhibitor of apoptosis in human hepatocellular carcinoma. Int J Mol Med 12: 705-708, 2003.

18. Vischioni B, van der Valk P, Span SW, Kruyt FA, Rodriguez JA and Giaccone G: Expression and localization of inhibitor of apoptosis proteins in normal human tissues. Hum Pathol 37: 78-86, 2006.

19. Leaman DW, Chawla-Sarkar M, Vyas K, Reheman M, Tamai K, Toji S and Borden EC: Identification of X-linked inhibitor of apoptosis-associated factor-1 as an interferon-stimulated gene that augments TRAIL Apo2L-induced apoptosis. J Biol Chem 277: 28504-28511, 2002.

20. $\mathrm{Ng} \mathrm{KC}$, Campos EI, Martinka M and Li G: XAF1 expression is significantly reduced in human melanoma. J Invest Dermatol 123: 1127-1134, 2004.

21. Ma TL, Ni PH, Zhong J, Tan JH, Qiao MM and Jiang SH: Low expression of XIAP-associated factor 1 in human colorectal cancers. Chin J Dig Dis 6: 10-14, 2005

22. Lee MG, Huh JS, Chung SK, et al: Promoter CpG hypermethylation and downregulation of XAF1 expression in human urogenital malignancies: implication for attenuated p53 response to apoptotic stresses. Oncogene 25: 5807-5822, 2006.

23. Pestka S, Langer JA, Zoon KC and Samuel CE: Interferons and their actions. Ann Rev Biochem 56: 727-777, 1987.

24. Gutterman JU: Cytokine therapeutics: lessons from interferon $\alpha$. Proc Natl Acad Sci USA 91: 1198-1205, 1994.

25. Yano H, Yanai Y, Momosaki S, et al: Growth inhibitory effects of interferon- $\alpha$ subtypes vary according to human liver cancer cell lines. J Gastroenterol Hepatol 21: 1720-1725, 2006.

26. Yano H, Ogasawara S, Momosaki S, et al: Growth inhibitory effects of pegylated IFN $\alpha-2 b$ on human liver cancer cells in vitro and in vivo. Liver Int 26: 964-975, 2006.

27. Yano H, Iemura A, Haramaki M, Ogasawara S, Takayama A, Akiba $\mathbf{J}$ and Kojiro M: Interferon alfa receptor expression and growth inhibition by interferon alfa in human liver cancer cell lines. Hepatology 29: 1708-1717, 1999.

28. Obi S, Yoshida H, Toune R, et al: Combination therapy of intraarterial 5-fluorouracil and systemic interferon-alpha for advanced hepatocellular carcinoma with portal venous invasion. Cancer 106: 1990-1997, 2006. 